\title{
Pre-hospital acute coronary syndrome management
}

EMS MONCA application

\section{G. Almeida1, M. Gusmão1, F. Matos², I. Fragata3}

${ }^{1}$ Anesthesiology resident, Centro Hospitalar de Lisboa Central, Lisbon, Portugal ${ }^{2}$ Anesthesiology graduated senior, Centro Hospitalar de Lisboa Central, Lisbon, Portugal

${ }^{3}$ Anesthesiology Head of Department, Centro Hospitalar de Lisboa Central, Lisbon, Portugal
Contacts

Goncalo.n.almeida@gmail.com

\section{Background}

\section{initial diagnostic suspicion infarction \\ pre hospital approach \\ risk assessment \\ ECG \\ transfer to the appropriate hospital center minimize delays}

coronary fasttrack protocol CFTP bypass normal ER referral shorten the time between first medical contact and reperfusion therapy

\section{Aim of study}

describe and characterize the initial approach and treatment of myocardial infarction patients referred to ER or CFTP

\section{Methods}

\section{Retrospective study}

patients diagnosed with acute coronary syndrome in the pre-hospital setting

\section{Apr 2014 - Nov 2015}

Time frame analyzed

\section{Collected data}

No-CFTP or CFTP

Gender

Age

Initial triage result

ECG performed

EMS treatment (Morphine, Oxygen, Nitrates, Clopidogrel and Acetylsalicylic acid)

Results

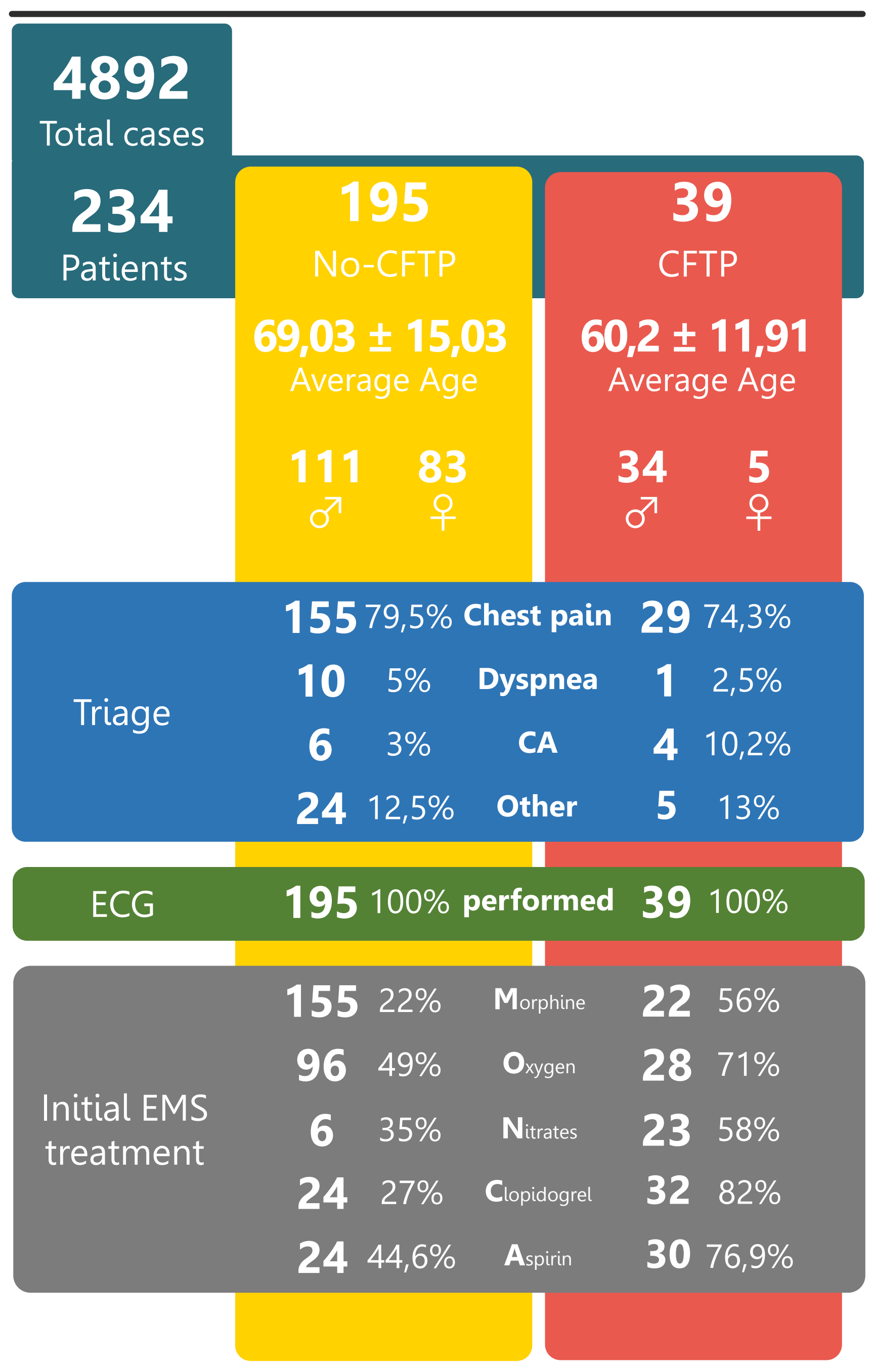

\section{Conclusions}

There are differences between patients in both groups, concerning initial approach and treatment.

The CFTP patients appear to be younger and their diagnosis and initial treatment appears to be started earlier than in patients in the no-CFTP group.

Optimization of care in cases of acute coronary syndrome can be achieved by better logistics, risk assessment, triage and early initiation of treatment. 